Acta Crystallographica Section D

Biological

Crystallography

ISSN 0907-4449

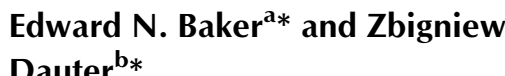

Dauter $^{b_{*}}$

${ }^{a}$ School of Biological Sciences, University of Auckland, Macromolecular, Private Bag 92-019, Auckland, New Zealand, and ${ }^{\mathbf{b}}$ Argonne National Laboratory, Biosciences Division, Bldg 202, Room Q142, Argonne, IL 60439, USA

\section{Small angle scattering - moving forward}

One of the great strengths of crystallographic research has always been the willingness of crystallographers to work together to develop standards that will enhance both the quality and the impact of our science. This attitude, facilitated by the International Union of Crystallography (IUCr) though its various Commissions, has led to advances that have benefited much wider constituencies. A good example is the policies developed, through the IUCr, relating to the deposition and release of biological structural data (Baker et al., 1996; Baker \& Saenger, 1999; Commission on Biological Macromolecules, 2000). The present issue of Acta Cryst. $D$ highlights another such initiative in a rapidly developing area of importance to structural biology: small-angle scattering (Jacques et al., 2012).

Small-angle scattering (SAS), whether of X-rays or neutrons, has been used for many years to gain structural insights into biological molecules in solution. One key advantage is obvious - you do not need crystals! In the past few years, a number of factors have come together to generate an explosion of interest in the use of SAS. New, high-quality, SAS beamlines have been developed at synchrotrons. Powerful and easy-to-use software has been developed for the interpretation of SAS data. As structural biologists tackle problems of everincreasing complexity, SAS data can be used in a variety of ways: to help interpret crystal structures by relating them to solution, to map substructures on to larger assemblies or to develop de novo information on large systems.

At the same time, there are potential hazards. SAS experiments are easy to perform, and with powerful, easy-to-use software available, models are easily generated. The question then becomes one of how valid the interpretations of a particular experiment may be. On p. 620 of this issue, David Jacques, Mitchell Guss, Dmitri Svergun and Jill Trewhella take a very important step forward. They present a set of guidelines that should go a long way towards ensuring that conclusions drawn from SAS experiments can be properly assessed by readers and reviewers. These guidelines, developed through the IUCr Commission on Small-angle Scattering, consider issues such as the nature and quality of the sample, the solvent used, SAS data collection and processing protocols, the parameterization of models and the validation of results. These guidelines and the associated discussion should be read and thought about by anyone planning an SAS experiment, and will undoubtedly be of great value to researchers as the use of SAS continues to expand.

It is also noteworthy that, in parallel, the Protein Data Bank has established an SAS Task Force to advise on whether models based on SAS analysis should be deposited, and, if so, with what accompanying data and what forms of validation. Meanwhile, the stage is set for continued expansion of this important technique. As Editors of a journal dedicated to biological structure and structural methods we welcome this initiative.

\section{References}

Baker, E. N., Blundell, T. L., Vijayan, M., Dodson, E., Gilliland, G. L. \& Sussman, J. L. (1996). Acta Cryst. D52, 609.

Baker, E. N. \& Saenger, W. (1999). Acta Cryst. D55, 2-3.

Commission on Biological Macromolecules (2000). Acta Cryst. D56, 2.

Jacques, D. A., Guss, J. M., Svergun, D. I. \& Trewhella, J. (2012). Acta Cryst. D68, 620-626. 